\title{
Assembly and characterization of fast neutron detectors for TREAT fuel motion monitoring system
}

\author{
Preston James Walker
}

August 2019

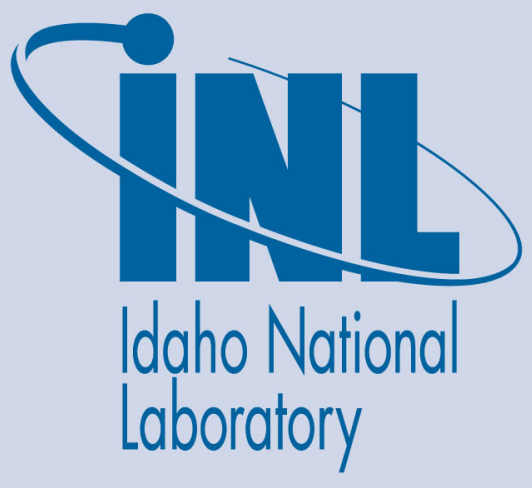

The INL is a U.S. Department of Energy National Laboratory operated by Battelle Energy Alliance 


\title{
Assembly and characterization of fast neutron detectors for TREAT fuel motion monitoring system
}

\author{
Preston James Walker
}

\author{
August 2019 \\ Idaho National Laboratory \\ Idaho Falls, Idaho 83415 \\ http://www.inl.gov \\ Prepared for the \\ U.S. Department of Energy \\ Under DOE Idaho Operations Office \\ Contract DE-AC07-05ID14517
}




\title{
Assembly and Characterization of Fast Neutron Detectors for TREAT Fuel Motion Monitoring System
}

\author{
Preston Walker, Idaho State University
}

\section{Background}

A key instrument supporting transient fuel testing at INL's Transient Reactor Facility (TREAT) is the Fuel Motion Monitoring System (FMMS) historically called the hodoscope. The FMMS measures the motion of fissionable material in a test capsule as the fuel fails under simulated accident-like conditions. The FMMS uses a large steel collimator with 360 slots that view the center of the reactor core. Original FMMS components, including detectors, data acquisition system, and electromechanical systems are 30-40 years old and haven't been used since the mid-1990s. They are all in need of repair, refurbishment, or replacement to support the TREAT restart program. To address this problem a comprehensive project is underway to restore the FMMS to full operational capability. To date, 96 of the original 360 channels have been restored and returned to operation at TREAT. An additional 96 channels are being readied at the Radiation Detection Systems Laboratory (RDSL) for deployment to TREAT in FY2020.

\section{Testing Methodology}

The purpose of this project was to qualify, assemble, and characterize 96 Proton Recoil Scintillator (PRS) detector assemblies. Each assembly consists of four main components: a photo-multiplier tube (PMT), a voltage divider, a PRS detector, and a Phenolic shroud. The PMT qualification and assembly characterization was completed at REC RDSL to achieve optimized operation before being deployed to the TREAT FMMS.

The first step in assembly was connecting a Hamamatsu R1166 PMT to a factory provided voltage divider which was then placed into a phenolic tube. An initial qualification test was performed using a pulsed $420 \mathrm{~nm}$ LED light source. The light source first required normalization to create a correction factor that could be applied to the incoming PMT signal. Once the correction was completed, each PMT was gain swept as a function of bias voltage to establish a characteristic gain response without a PRS detector attached. Next, the same gain sweep was performed without the LED light source to measure dark current. After those parameters were established, remanufactured PRS detectors were attached to the PMTs using index of refraction matching optical gel to improve light transmission between PMT and PRS. The subassemblies were aligned and secured in a phenolic tubes. The complete PRS assembly characterization was performed using three low activity ${ }^{252} \mathrm{CF}$ sources. The ${ }^{252} \mathrm{CF}$ sources also needed to be normalized due to non-Gaussian neutron distribution with respect to source to detector distance because multiple sources were used to obtain higher neutron count rates to reduce test times. This was preformed following the same methodology as the light source normalization. Final testing for PRS detector assemblies involved mapping the pulse shape discrimination using a figure of merit (FOM) bench mark and ensuring each assembly meets a predetermined signal to noise ratio.


Two lightproof enclosures which qualify 8 PMT's at a time using a normalized $420 \mathrm{~nm}$ LED light source (left) and test 8 PRS detector assemblies at a time using normalized ${ }^{252} \mathrm{Cf}$ sources (right).

Light Intensity vs Position
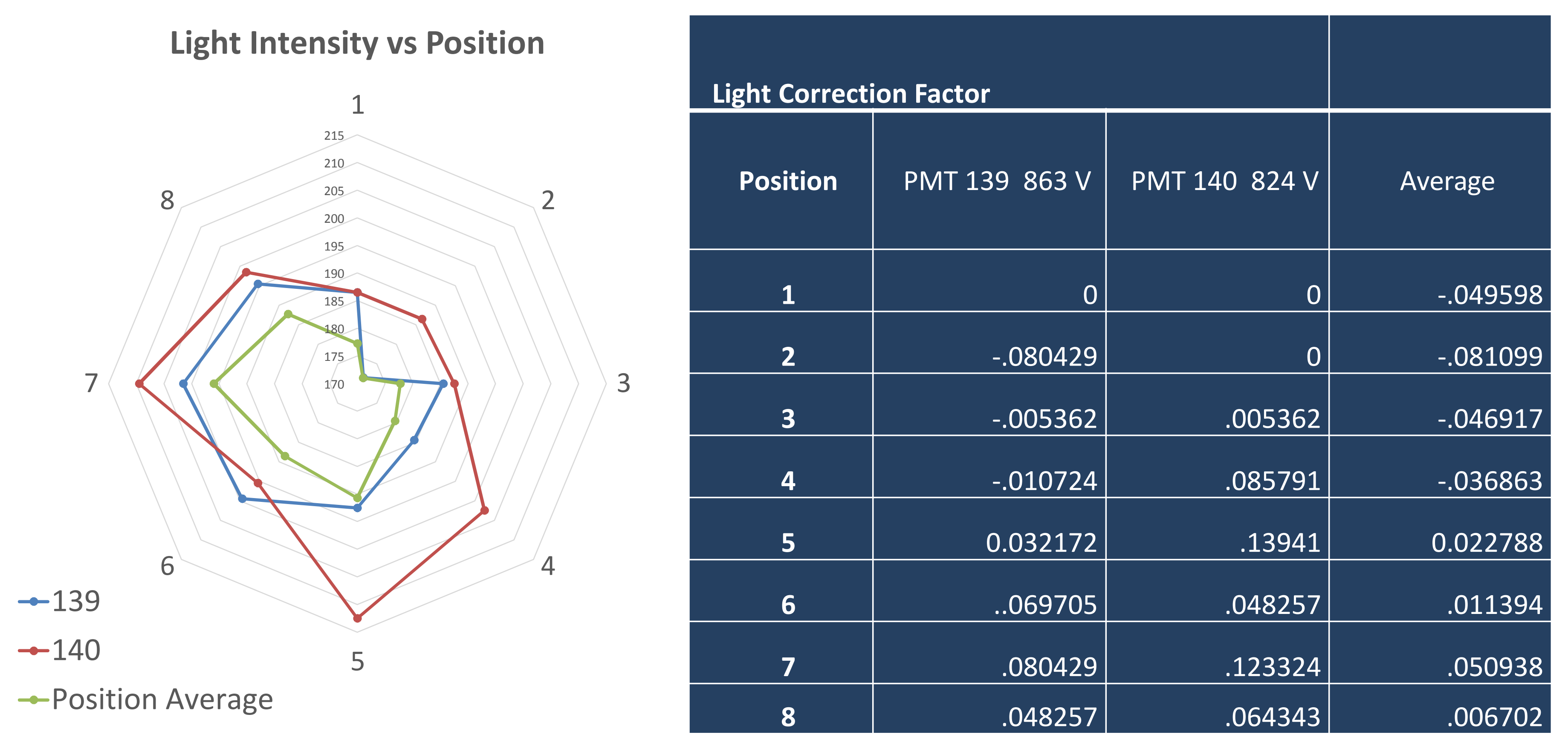

Computer controlled high voltage supplies were used to sweep PMT bias voltages while pulsing a normalized 420nm LED that was directly coupled to an acrylic light guide. Radial graph and normalization correction factors are shown above

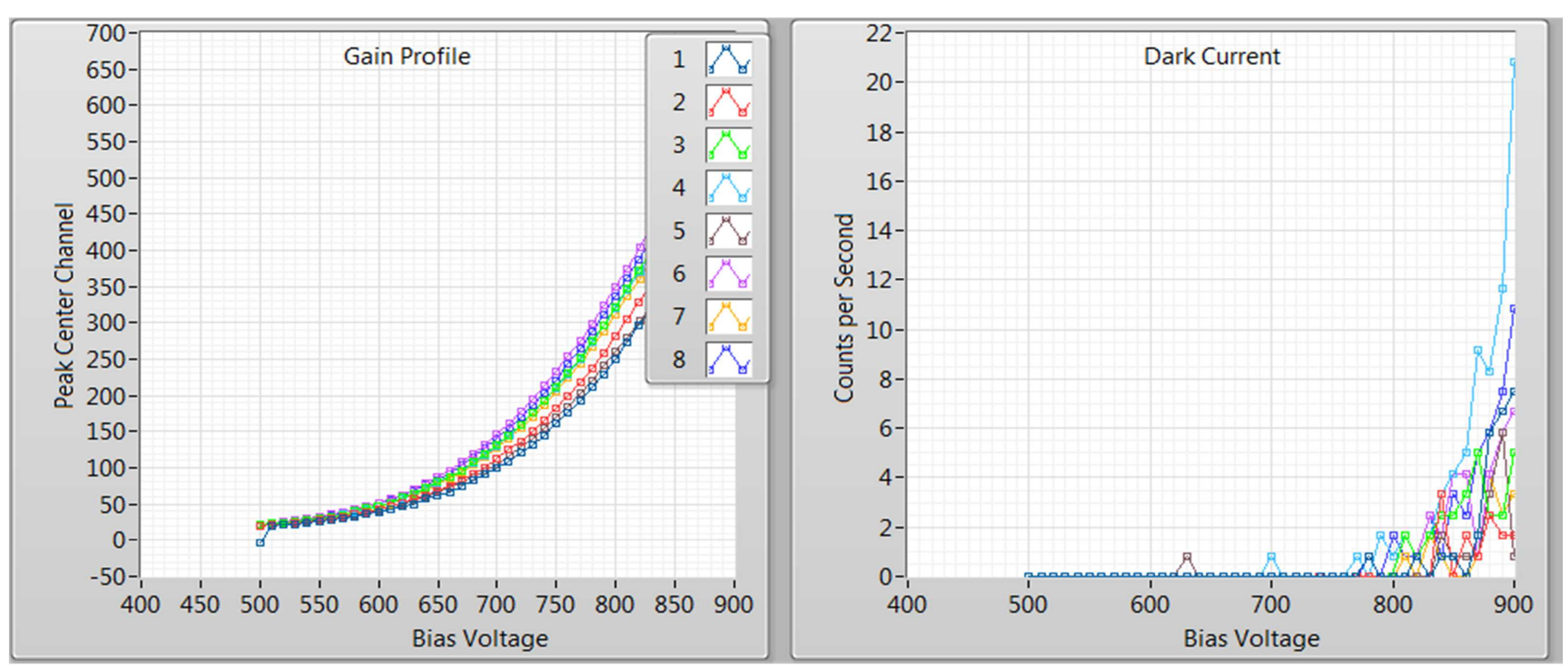

Graphs showing the concurrent gain profiles and dark current results of 8 individual PMT's under test

\section{Acknowledgements}

I would like to acknowledge and thank the TREAT Experiment Program for funding this internship opportunity. Thanks also to Mr. Scott Watson, Dr. David Chichester, Mr. James Johnson, and Mr. Jay Hix, for their mentorship and guidance on this project. 